\title{
SATISFACCIÓN CON LA VIDA EN GRUPOS ETARIOS DE LA CIUDAD DE AREQUIPA, PERÚ
}

\author{
Calizaya López José Manuel', Monzón Alvarez Gloria Isabel' ${ }^{2}$, Miaury Vilca Ana Rosario ${ }^{3}$, \\ Pinto Pomareda Hilda Lizbeth ${ }^{4}$ y Evangelista Aliaga José Luis ${ }^{5}$ \\ jcalizayal@unsa.edu.pe ${ }^{1}$,gmonzon@unsa.edu.pe ${ }^{2}$, amiaury@unsa.edu.pe ${ }^{3}$, \\ hpintop@unsa.edu.pe ${ }^{4}$,jevangelista@unsa.edu.pe ${ }^{5}$ \\ https://orcid.org/0000-0001-6221-09091, https://orcid.org/0000-0001-8712-51882, \\ https://orcid.org/0000-0002-2992-12393, https://orcid.org/0000-0002-1719-48634, \\ https://orcid.org/0000-0002-7749-31445 \\ Universidad Nacional de San Agustín de Arequipa \\ Arequipa - Perú
}

Recibido (09/10/20), Aceptado (28/11/20)

\begin{abstract}
Resumen: El objetivo del estudio fue analizar el nivel de satisfacción con la vida en grupos etarios según variables sociodemográficas, se realizó en 1661 sujetos (adolescentes, jóvenes, adultos y adultos mayores) de 3 distritos de la ciudad de Arequipa, a quienes se les aplicó el cuestionario de satisfacción con la vida. Se encontró que el nivel de satisfacción de los participantes es moderado con tendencia a ser alta. Según grupo etario, los adultos mayores presentan niveles altos de satisfacción con la vida en comparación a los otros grupos y las personas que presentan mayor nivel educativo se encuentran más satisfechos con la vida. Se concluye que, los resultados guardan relación con el nivel de bienestar y calidad de vida que experimentan las personas haciendo una evaluación global positiva de su experiencia en la vida.
\end{abstract}

Palabras Clave: Satisfacción con la vida, grupos etarios, variables sociodemográficas.

\section{SATISFACTION WITH LIFE IN AGE GROUPS IN THE CITY FROM AREQUIPA, PERU}

\begin{abstract}
The objective of the study was to analyze the level of satisfaction with life in age groups according to sociodemographic variables, it was carried out in 1661 subjects (adolescents, youth, adults and older adults) from 3 districts of the city from Arequipa, to whom the life satisfaction questionnaire. It was found that the level of satisfaction of the participants is moderate with a tendency to be high. According to age group, older adults have high levels of satisfaction with life compared to other groups and people with a higher educational level are more satisfied with life. It is concluded that the results are related to the level of well-being and quality of life that people experience by making a positive global evaluation of their experience in life.
\end{abstract}

Keywords: Satisfaction with life, age groups, sociodemographic variables. 


\section{INTRODUCCIÓN}

En los últimos años el Perú tuvo indicadores de crecimiento económico importantes disminuyendo la pobreza monetaria [1], sin embargo, este incremento no se ve reflejado en la calidad de vida del ciudadano debido a que algunos indicadores de bienestar material como el acceso a la educación y salud aún no se han garantizado a toda la población, el trabajo se incrementó pero se redujo el empleo (informalidad), si bien las viviendas ya cuentan con servicios básicos se evidencia la precariedad en la construcción y sobre todo en la cantidad de personas que la habitan en reducidos espacios, a ello se suma que la inseguridad ciudadana es un problema central que genera miedo, estos aspectos también son valorados por los ciudadanos desde su ser interior determinado lo positivo o negativo de vivir en este país.

El Estado Peruano a través de las políticas públicas es el ente encargado de mejorar el bienestar de la población [2] con la finalidad de incrementar la calidad de vida del ciudadano accediendo a un bienestar tanto material como subjetivo, situación que aún sigue siendo una preocupación desatendida por parte de los gobiernos de turno [3]. Al respecto, las decisiones políticas se inclinan a favorecer a unos cuantos, generando altos niveles de corrupción y bajos niveles de atención a las necesidades básicas de la población, indicadores que probablemente puede incidir en la satisfacción vital de los ciudadanos.

En los últimos años la psicología positiva [4] viene haciendo aportes sobre las experiencias reales que experimentan las personas para establecer que los hace felices y que no, indagando aspectos que están relacionados al bienestar y la calidad de vida, con la finalidad de prevenir o reducir la incidencia de la psicopatología [5].

En tal sentido, la satisfacción con la vida está asociada al nivel de bienestar subjetivo y material que experimentan las personas en su vida cotidiana [6] aspectos relacionados al trabajo, estudios, familia, amigos que implican sentimientos y estados de ánimo diferentes. Al respecto, según Arias, Huamani y Caycho [7] precisan que el nivel de evaluación individual que realiza cada persona sobre su calidad de vida global se denomina satisfacción con la vida, considerándose criterios subjetivos sobre aspectos físicos, sociales y mentales [8].

Es decir, a partir de las experiencias cotidianas tanto positivas como negativas en los diferentes espacios de socialización la persona determina el nivel de satisfacción que encuentra en su vida a nivel psicológico, fisiológico, espiritual, biológico, cultural y material.

Por lo tanto, el objetivo del estudio fue analizar el nivel de satisfacción con la vida en grupos etarios según variables sociodemográficas en Arequipa. El estu- dio es transversal, retrospectivo, expost facto, además, el tiempo de recolección de información correspondió a los meses de diciembre 2019 y enero-febrero del año 2020 .

\section{DESARROLLO}

La satisfacción vital está íntegramente relacionada a la de calidad de vida y es utilizada indistintamente en muchos campos sobre todo en el político y académico encontrando contradicciones sobre todo por el uso que se le da, sin embargo, la calidad de vida está asociada a las condiciones que se brinda a una persona para ser agradable y valiosa su vida encontrado niveles de bienestar que le permiten disfrutar o no de ella [9].

En ese sentido, al hacer referencia al bienestar se explica que es un estado individual y colectivo para demostrar capacidad frente a situaciones adversas o no de la vida que experimentan las personas, en la medida que se superen se incrementa el nivel de bienestar [10] además, el bienestar difiere según las etapas de vida dada las necesidades y expectativas en cada proceso vivencial [11].

Según Murillo y Molero [12] explican que el nivel de bienestar depende de las necesidades satisfechas (subjetivas y objetivas), por lo que, el bienestar subjetivo se caracteriza por las emociones o también felicidad [13] a través de las opiniones favorables o desfavorables de los individuos y es el resultado de la satisfacción vital [14]. Y en relación con el bienestar objetivo se consideran las condiciones externas al sujeto como: ingresos económicos, vivienda, acceso a servicios de salud y educación de calidad, así como, un adecuado nivel de recreación [15].

Además, la satisfacción vital ha sido analizada y asociada a variables psicosociales destacando las más relevantes el apoyo social, autoestima, satisfacción laboral, identidad, migración, bienestar material, así como, variables sociodemográficas (edad, sexo, estado civil, lugar de residencia, nivel educativo) considerándolas como variables predictivas de la satisfacción con la vida [16].

Por lo que, la mayoría de estudios relacionados al tema de investigación propuesto se han centrado en evaluar el grado de satisfacción vital dentro de los grupos etarios más en adolescentes y adultos mayores que en jóvenes y adultos, al respecto, algunos investigadores [17] [18] demostraron alta satisfacción con la vida en general en muestras de adolescentes no encontrando diferencias por el tipo de institución educativa, así también, los adultos mayores presentaron niveles elevados de satisfacción vital [19] encontrando diferencias esta- 
dísticamente significativas en relación al sexo (adulto mayor mujer experimenta mejor satisfacción que el adulto mayor varón), señalando que las mujeres presentan menos problemas de salud que los varones, no obstante, para Inga y Vara [20] si bien las adultas mayores presentan mejores niveles de satisfacción vital son las que también experimentan soledad y abandono producto de la condición civil de la viudez.

Sin embargo, algunos estudios [21] [22] demostraron que las evidencias científicas sobre satisfacción vital en jóvenes (universitarios), están relacionados a la satisfacción con los saberes incidiendo que la formación académica es un factor de satisfacción vital que experimenta el estudiante universitario al concluir su etapa de formación. Al igual, el adulto encuentra satisfacción vital cuando se desarrolla dentro de un ambiente laboral adecuado de prestigio y es reconocido moral y materialmente por la organización [23].

Por lo tanto, el estudio de la satisfacción con la vida es relevante para las diferentes disciplinas que estudian el comportamiento humano y social, para identificar las expectativas, necesidades y sobre todo la valoración de la existencia según grupos etarios.

\section{III.METODOLOGÍA}

Se incluyeron 1661 sujetos (449 adolescentes de 12 a 17 años, 587 jóvenes de 18 a 29 años, 567 adultos de 30 a 59 años y 58 adultos mayores de 60 años a más) de 3 distritos de la ciudad de Arequipa, quienes fueron seleccionados aleatoriamente [24]. El 50\% fueron hombres y el otro $50 \%$ mujeres. $53.7 \%$ fueron estudiantes, 6.2\% amas de casa, 20.3\% trabajadores(as) independientes, $18.8 \%$ trabajadores(as) dependientes y el $1 \%$ desempleados. $59.5 \%$ su estado civil fue de soltero(a), $19.1 \%$ casado(a), $1 \%$ viudo(a), $0.7 \%$ divorciado(a) y $19.7 \%$ conviviente. $\mathrm{Y}$ según su nivel educativo $3.6 \%$ contaban con primaria, $43.9 \%$ secundaria y $52.6 \%$ nivel educativo superior.

Se aplicó la escala de satisfacción con la vida [25] adaptado y traducido [26], el instrumento consta de 5 ítems que evalúan el nivel de satisfacción vital a través del juicio global que hacen las personas sobre esta, en esta versión se llevó a cabo una reducción en las opciones de respuesta del instrumento, los valores se encuentran entre 1 y 5 de respuesta tipo Likert donde 1 es "totalmente en desacuerdo" y 5 es "totalmente de acuerdo", la puntuación total de la escala va de 5 (baja satisfacción) a 25 (alta satisfacción). Así mismo, en el instrumento se adicionaron características sociodemográficas como edad, sexo, estado civil, ocupación y grado de instrucción, además se consignaron los datos del consentimiento informado. Para la versión adaptada a la muestra local se obtuvieron los niveles de confiabilidad de la escala satisfacción con la vida mediante el método de consistencia interna y con la prueba alfa de Cronbach, por lo tanto, el instrumento posee buena confiabilidad cuando los valores (alfa) $\alpha \geq 0.700$ [27] para el caso se obtuvo un $\alpha=0.773$ de acuerdo con la medición el alfa es aceptable.

Para la aplicación del instrumento se obtuvo asentimiento y consentimiento de los adolescentes, previa autorización de sus centros educativos y padres, se incluyeron personas mayores que vivían en la comunidad, quienes fueron contactadas, explicando el objetivo del estudio y obteniendo consentimiento informado. Posteriormente se entregó el instrumento para que cada grupo respondiera de manera individual.

\section{RESULTADOS}

Se exploraron los datos para analizar la distribución, la asimetría, curtosis y pruebas de normalidad evidenciando que no existió distribución normal $(\mathrm{p}<$ .05). Se realizó el análisis descriptivo y el comparativo de la satisfacción con la vida según sexo, grupo etario, ocupación, estado civil y grado de instrucción. Para comparar dos muestras independientes se utilizó la U de Mann-Whitney con su respectivo tamaño del efecto (TE) calculó de la probabilidad de superioridad (PSest) las normas interpretativas son no efecto (PSest $\leq=0.0$ ), pequeño (PSest $\geq 0,56$ ), mediano (PSest $\geq 0,64$ ) y grande (PSest $\geq 0,71)$ [28]. La comparación de más de dos muestras independientes se realizó con la $\mathrm{H}$ de Kruskal Wallis su tamaño del efecto utilizado fue $(\mathrm{n} 2 \mathrm{H})$ eta cuadrado [29] siendo sus normas interpretativas (n2H $\geq$ 0.04 mínima necesaria), ( $\mathrm{n} 2 \mathrm{H} \geq 0.25$ moderada), ( $\mathrm{n} 2 \mathrm{H}$ $\geq 0.64$ fuerte) [30] y para establecer las correlaciones se empleó la Rho de Spearman. 
TABLA I. Valores numéricos del nivel de satisfacción con la vida

\begin{tabular}{cccccc}
\hline Nivel & $M$ & $D E$ & $M d$ & Min. & Máx. \\
\hline Satisfacción con la vida & 17.10 & 4.08 & 17 & 5 & 25 \\
\hline
\end{tabular}

$\mathrm{M}=$ Media; $\mathrm{D}=$ Desviación estándar; $\mathrm{Md}=$ Mediana; Min. = mínimo; Máx. = máximo

En la tabla se describen los resultados de los participantes en la escala de Diener, el nivel de satisfacción con la vida de acuerdo con la media es de 17.1 con una desvia- ción estándar de 4.08 , de un puntaje máximo de 25 , por lo tanto, el nivel de satisfacción de los participantes es moderado con tendencia a una alta satisfacción.

TABLA II. Nivel de satisfacción con la vida según grupos etarios

\begin{tabular}{ccccccccc}
\hline & \multicolumn{2}{c}{ Adolescente } & \multicolumn{2}{c}{ Joven } & \multicolumn{2}{c}{ Adulto } & \multicolumn{2}{c}{ Adulto Mayor } \\
Nivel & $($ de 12 a 17 años $)$ & \multicolumn{2}{c}{ (de 18 a 29 años) } & (de 30 a 59 años) & \multicolumn{2}{c}{ (más de 60 años) } \\
& $f(x)$ & $\%$ & $f(x)$ & $\%$ & $f(x)$ & $\%$ & $f(x)$ & $\%$ \\
\hline Baja & 205 & $45.7 \%$ & 18 & $3.1 \%$ & 3 & $0.5 \%$ & 0 & $0.0 \%$ \\
Moderada & 240 & $53.5 \%$ & 499 & $85.0 \%$ & 215 & $37.9 \%$ & 0 & $0.0 \%$ \\
Alta & 4 & $0.9 \%$ & 70 & $11.9 \%$ & 349 & $61.6 \%$ & 58 & $100.0 \%$ \\
Total & 449 & $100 \%$ & 587 & $100 \%$ & 567 & $100 \%$ & 58 & $100 \%$ \\
\hline
\end{tabular}

Nota: $\mathrm{f}(\mathrm{x})=$ frecuencia; $\%=$ porcentaje; $\mathrm{X} 2=$ Chi-cuadrado; $\mathrm{p}=\mathrm{p}$ valor

En cuanto a la satisfacción con la vida según grupos etarios se identifica, en el grupo de adolescentes para el $53.5 \%$ el nivel es moderado con una tendencia a ser baja, debido a que de acuerdo con la edad adolecen de aspectos emocionales buscando independencia sobre todo perciben que un aspecto que los frustra es la escaza autonomía y las dificultades que encuentran en la interacción social en sus espacios de socialización siendo en varias oportunidades problemático y conflictivo. En los jóvenes el nivel de satisfacción es de $85 \%$ moderado, siendo categorizado como parcialmente satisfecho, esto se debe a situaciones que aún les falta experimentar como la autonomía, el crecimiento personal, el cumplimento de metas y objetivos, la realización afectiva con la pareja, la valoración de lo conseguido y la consolidación laboral.

En los adultos el $61.6 \%$ de satisfacción vital es alto debido a que sus experiencias en la vida tanto positivas como negativas han servido de valoración precisando que sus experiencias laborales los han desarrollado profesionalmente.

Y en el adulto mayor el $100 \%$ indica que se siente altamente satisfecho con la vida, debido a que su experiencia de vida sobre todo el relacionado a la familia ha sido valorado positivamente y los han realizado como seres humanos.

TABLA III. Comparación de la satisfacción con la vida según variables sociodemográficas

\begin{tabular}{|c|c|c|c|}
\hline & $\begin{array}{c}\text { Grupo etario } \\
\text { categoría (n) } \\
\text { Rango promedio }\end{array}$ & $\begin{array}{c}\text { Ocupación } \\
\text { categoría }(\mathrm{n}) \\
\text { Rango promedio }\end{array}$ & $\begin{array}{c}\text { Estado civil } \\
\text { categoría (n) } \\
\text { Rango promedio }\end{array}$ \\
\hline \multirow{6}{*}{ Satisfacción con la vida } & $\begin{array}{c}\text { Adolescencia } \\
\quad(n=449) \\
440.52\end{array}$ & $\begin{array}{c}\text { Estudiante } \\
(n=892) \\
605.82\end{array}$ & $\begin{array}{c}\text { Soltero/a } \\
\begin{array}{c}(n=989) \\
610.96\end{array}\end{array}$ \\
\hline & $\begin{array}{c}\text { Juventud } \\
(n=587) \\
770.97\end{array}$ & $\begin{array}{c}\text { Ama de casa } \\
\left(\begin{array}{l}(n=103) \\
1156.29\end{array}\right.\end{array}$ & $\begin{array}{c}\text { Casado/a } \\
(n=317) \\
1144.21\end{array}$ \\
\hline & $\begin{array}{c}\text { Adultez } \\
(n=567) \\
1142.01 \\
\end{array}$ & $\begin{array}{c}\text { Trabajador(a) independiente } \\
\qquad \begin{array}{c}(n=337) \\
1093.50\end{array} \\
\end{array}$ & $\begin{array}{l}\text { Viudo/a } \\
(n=16) \\
1331.31 \\
\end{array}$ \\
\hline & \multirow{2}{*}{$\begin{array}{l}\text { Adulto Mayor } \\
\qquad \begin{array}{l}(n=58) \\
1421.0\end{array}\end{array}$} & $\begin{array}{c}\text { Trabajador(a) dependiente } \\
\qquad(n=312) \\
1167.76\end{array}$ & $\begin{array}{c}\text { Divorciado/a } \\
(n=11) \\
1225.32\end{array}$ \\
\hline & & $\begin{array}{c}\text { Desempleado } \\
(n=17) \\
1065.52\end{array}$ & $\begin{array}{c}\text { Conviviente } \\
(n=328) \\
1154.13\end{array}$ \\
\hline & $\begin{array}{c}H=807.638 \\
P=, 000 \\
n^{2} H=0.49\end{array}$ & $\begin{array}{c}H=545.985 \\
P=, 000 \\
n^{2}=0.33\end{array}$ & $\begin{array}{c}H=659.709 \\
P=, 000 \\
n^{2}{ }_{H}=0.40\end{array}$ \\
\hline
\end{tabular}

Nota: $\mathrm{n}=$ tamaño muestral; $\mathrm{H}=\mathrm{H}$ de Kruskal Wallis; $\mathrm{p}=\mathrm{p}$ valor. $\mathrm{n} 2 \mathrm{H}=$ eta cuadrada (tamaño del efecto). 
En la tabla se compararon los niveles de satisfacción con la vida con variables sociodemográficas, encontrando los siguientes resultados: En relación con el grupo etario, se encontraron diferencias estadísticamente significativas siendo los adultos mayores quienes presentan niveles altos de satisfacción con la vida en comparación a los otros grupos (tamaño del efecto moderado $\mathrm{n} 2 \mathrm{H}=0.49$ ), ratificando los resultados descriptivos categóricos de la tabla I.

En cuanto a la ocupación, también se encontraron diferencias significativas siendo los trabajadores(as) dependientes con más altos niveles de satisfacción con la vida (tamaño del efecto moderado $\mathrm{n} 2 \mathrm{H}=0.33$ ), al respecto, la ocupación sobre todo cuando se tiene la condición de dependencia laboral para una organización influye en el nivel de satisfacción vital, permite el crecimiento per- sonal y profesional, se aseguran los ingresos económicos para cubrir las necesidades familiares, se fortalece la autonomía y responsabilidad, es reconocido moral y materialmente por la organización.

Así mismo, de acuerdo con el estado civil de los participantes se encontraron diferencias significativas, siendo los viudos(as), quienes se encuentran más satisfechos con la vida (tamaño del efecto moderado $\mathrm{n} 2 \mathrm{H}=0.40$ ), las personas en condición de viudez son adultos mayores que a pesar de la soledad y abandono resultado de la muerte de la pareja, es la familia quien alberga a esta población, y en esta etapa de vida por lo general, los adultos mayores ayudan en la crianza de los nietos situación que los lleva a satisfacer su vida emocional al sentirse importantes en la formación de sus descendientes.

TABLA IV. Comparación de la satisfacción con la vida según sexo

\begin{tabular}{lccccccc}
\hline \multirow{2}{*}{ Nivel } & \multicolumn{2}{c}{$\begin{array}{c}\text { Hombres } \\
(n=831)\end{array}$} & \multicolumn{2}{c}{$\begin{array}{c}\text { Mujeres } \\
(n=830)\end{array}$} & \multicolumn{2}{c}{ Estadigrafo } & \multirow{2}{*}{$P S_{\text {est }}$} \\
\cline { 2 - 6 } & $M(D E)$ & $M e$ & $M(D E)$ & $M e$ & $U$ & $p$ & \\
\hline $\begin{array}{l}\text { Satisfacción } \\
\text { con la vida }\end{array}$ & $17.17(3.85)$ & 17 & $17.03(4.30)$ & 17 & 344824.0 & 0,997 & 0.49 \\
\hline
\end{tabular}

Nota: $\mathrm{n}=$ tamaño muestral; $\mathrm{M}=$ Media; $\mathrm{Me}=$ Mediana; $\mathrm{DE}=$ Desviación estándar; $\mathrm{U}=\mathrm{U}$ de Mann Withney; $\mathrm{p}=$ $\mathrm{p}$ valor; PSest= Probabilidad de Superioridad (tamaño del efecto).

Se compararon los niveles de satisfacción con la vida en hombres y mujeres, encontrándose que no existen diferencias estadísticamente significativas, siendo su tamaño del efecto pequeño, ambos sexos muestran similares niveles de satisfacción, es decir, se sienten parcialmente satisfechos sobre todo en la autoaceptación, en los propósitos de la vida y las relaciones amicales positivas.

Tabla V. Correlaciones de la satisfacción con la vida, la edad, y el grado de instrucción

\begin{tabular}{lccc}
\hline & Edad & Grado de instrucción & $\begin{array}{c}\text { Satisfacción con la } \\
\text { vida }\end{array}$ \\
\hline Edad & 1 & 1 & \\
Grado de instrucción & & $0,282 * *$ & 1 \\
Satisfacción con la vida & $0,760 * *$ & \\
\hline
\end{tabular}

**. La correlación es significativa en el nivel 0,01 ( 2 colas).

A nivel correlacional, se encontraron evidencias estadísticamente significativas en la edad y la satisfacción con la vida (correlación positiva, nivel alto ,760**) demostrándose que a mayor edad existe mayor nivel de satisfacción con la vida. Así también, ocurre en el grado de instrucción y la satisfacción con la vida (correlación positiva, nivel bajo ,760**) demostrándose que a mayor nivel educativo existe mayor nivel de satisfacción vital, la educación a través de la formación académica es un factor de satisfacción vital que experimentan las personas encontrando complacencia y expectativas de vida.

\section{V.CONCLUSIONES}

En el presente estudio después de evaluar a los gru- 
pos por etapas de vida (grupos etarios) se encontró que el nivel de satisfacción con la vida en general es moderado con una tendencia a ser alta satisfacción, estos resultados guardan relación con el nivel de bienestar y calidad de vida que experimentan las personas haciendo una evaluación global positiva de su experiencia en la vida.

Los adultos mayores presentan niveles altos de satisfacción con la vida en comparación a los otros grupos sobre todo en los adolescentes, precisándose que la experiencia de vida influye en la satisfacción vital, es decir a mayor edad mayor satisfacción.

En cuanto a la ocupación, los trabajadores(as) dependientes muestran altos niveles de satisfacción con la vida en comparación a las amas de casa, trabajadores independientes, desempleados y estudiantes, es decir, que un factor importante en la satisfacción vital es el desarrollo en el trabajo, explicando que en la etapa de la adultez se logra desarrollar este nivel de satisfacción cuando la persona encuentra un ambiente laboral adecuado y de prestigio siendo reconocido moral y materialmente por la organización.

De acuerdo con el estado civil los viudos(as) se encuentran más satisfechos con la vida, en comparación que los divorciados, casados, convivientes y solteros, al respecto, la pérdida del ser querido influye en el estado de ánimo de la persona, pero también se puede explicar que las personas encuestadas presentarían niveles altos de resiliencia.

El estudio es un precedente importante debido a que la satisfacción con la vida según grupos etarios difiere según la etapa de vida, diferenciándose el nivel de bienestar subjetivo y objetivo que experimentan las personas de acuerdo a sus expectativas, necesidades, experiencias que lógicamente sucede a lo largo de la vida de las personas, por ello se espera que el estudio haya sido de contribución académica para las disciplinas relacionadas al comportamiento humano y social como: la psicología, sociología, economía y las disciplinas que se interesan en el tema propuesto.

Finalmente, es relevante seguir estudiando la satisfacción con la vida en las diferentes esferas sociales tanto productivas, académicas, comunitarias y familiares, con la finalidad de tener mejores lineamientos sobre las experiencias de vida obteniendo una amplia información para comprender la vida subjetiva y objetiva de las personas.

\section{REFERENCIAS}

[1]J. Zanabria, «Crecimiento económico permitió disminuir la pobreza monetaria,» El Peruano, p. 3, 411 2019.
[2]C. Cómez, G. J. Suárez, J. E. Garzón y J. A. Gómez, «El ser y el tener de los habitantes de la ciudad de Medellín como determinantes de la satisfacción con la vida.,» Revista Latinoamericana de Desarrollo Económico, vol. 1, no 26, pp. 69-98, 2016.

[3]M. Rojas, «Economía de la felicidad. Hallazgos relevantes respecto al ingreso y el bienestar,» El Trimestre Económico, vol. 76, nº 303, pp. 537-573, 2009.

[4]M. Seligman y M. Csikszentmuhalyi, «Positive Psychology: An introduction,» American Psychologist, $\mathrm{n}^{\circ}$ 55, pp. 5-14, 2000.

[5]M. F. Rabito y J. M. Rodríguez, «Satisfacción con la vida y bienestar psicológico en personas con Disforia de Género,» Actas Especiales Psiquiatría, vol. 44, n 2 , pp. 47-54, 2016.

[6]J. Alfaro, J. Guzmán, F. Reyes, C. García, J. Varela y D. Sirlopú, «Satisfacción global con la vida y satisfacción escolar en estudiantes Chilenos,» Psykhe (Santiago), vol. 25, no 2, pp. 1-14, 2016.

[7]W. L. Arias, J. C. Huamani y T. Caycho, «Satisfacción con la vida en escolares de la ciudad de Arequipa,» Propósitos y Representaciones, vol. 6, $\mathrm{n}^{\mathrm{o}} 1$, pp. 351407, 2018.

[8]R. Moreta, C. López, P. Gordón, W. Ortíz y I. Gaibor, «Satisfacción con la vida, bienestar psicológico y social como predictores de la salud mental en ecuatorianos,» Actualidades en Psicología, vol. 32, n 124, pp. 112126, 2018.

[9]J. A. Fernández, M. Fernández y A. Cieza, «Los conceptos de calidad de vida, salud y bienestar analizados desde la perspectiva de la Clasificación Internacional del Funcionamiento (CIF),» Revista Española de Salud Pública, vol. 84, nº 2, pp. 169-184, 2010.

[10]J. García, «Psicología positiva, bienestar y calidad de vida,» En-claves del pensamiento, vol. 8, no 16, pp. 13-29, 2014.

[11]T. Mayodormo, A. Sales, E. Satorres y J. Meléndez, «Bienestar psicológico en función de la etapa de vida, el sexo y su interacción,» Pensamiento Psicológico, vol. 14, $n^{\circ}$ 2, pp. 101-112, 2016.

[12]J. Murillo y F. Molero, «La Satisfacción Vital: Su relación con el prejuicio, la identidad nacional, la autoestima y el bienestar material, en inmigrantes, $\gg$ Acta Colombiana de Psicología, vol. 15, n 2, pp. 99-108, 2012.

[13]J. Martell, C. H. García, L. Daniel, P. Sánchez y A. Mendoza, «Estructura factorial de la escala de satisfacción con la vida y validez convergente con la escala de calidad de vida en preparatorianos mexicanos,» Revista de psicología y ciencias del comportamiento de la Unidad Académica de Ciencias Jurídicas y Sociales, vol. 9, $\mathrm{n}^{\mathrm{o}} 2$, pp. 30-45, 2018. 
[14]E. Calvo y P. Beytía, «¿Cómo medir la felicidad?,» Claves de Políticas Públicas, vol. 1, nº 4, pp. 1-11, 2011. [15]M. Vallejo y M. d. P. Moreno, «Satisfacción vital y su relación con otras variables psicosociales en población española residente en Alemania,» Escritos de Psicología, vol. 9, nº 2, pp. 12-21, 2016.

[16]N. Pérez y A. Alegre, «Satisfacción con la vida: predictores y moderadores.,» Psicologia positiva i benestar, vol. 1, n⿳0 1, pp. 447-457, 2014.

[17]C. A. Alvarez, A. M. Briceño, K. Álvarez, M. Abufhele y I. Delgado, «Estudio de adaptación y validación transcultural de una escala de satisfacción con la vida para adolescentes,» Revista Chilena de Pediatría, vol. 89, no 1, pp. 51-58, 2018.

[18]E. López, N. Pérez y A. Alegre, «Competencia emocional, satisfacción en contextos específicos y satisfacción con la vida en la adolescencia,» Revista de Investigación Educativa, vol. 36, nº 1, pp. 57-73, 2018. [19]M. Ramírez y S.-L. Lee, «Factores asociados a la satisfacción vital en adultos mayores de 60 años,» Polis (Santiago), vol. 11, no 33, pp. 407-428, 2012.

[20]J. Inga y A. Vara, «Factores asociados a la satisfacción de vida de adultos mayores de 60 años en Lima-Perú,» Universidad Psychology Bogotá (Colombia), vol. 5, no 3, pp. 475-485, 2006.

[21]P. Ruiz, Y. Medina, A. Zayas y R. Gómez, «Relación entre la autoestima y la satisfacción con la vida en una muestra de estudiantes universitarios,» Revista INFAD de Psicología, vol. 2, nº 1, pp. 67-76, 2018.

[22]C. Merino, S. Domínguez y M. Fernández, «Validación inicial de una Escala Breve de Satisfacción con los Estudios en estudiantes universitarios de Lima,» Educación Médica, vol. 18, nº 1, pp. 74-77, 2017.

[23]J. D. Polo, M. Fernández y R. G. Ramírez, «Diseño del trabajo y satisfacción con la vida,» Revista Venezolana de Gerencia, vol. 17, nº 59, pp. 466-481, 2012. [24]S. Carrasco, Metodología de la Investigación Científica. Pautas metodológicas para diseñar y elaborar el proyecto de investigación, Lima: Editorial San Marcos, 2019.

[25]E. Diener, R. A. Emmons, R. J. Larsen y S. Griffin, «The Satisfaction with Life Scale.,» Journal of Personality Assessment, vol. 49, no 1, pp. 71-75, 1985.

[26]F. L. Atienza, D. Pons, I. Balaguer y M. García, «Propiedades psicométricas de la Escala de Satisfacción con la Vida en adolescentes,» Psicothema, vol. 12, $\mathrm{n}^{\circ}$ 2, pp. 314-319, 2000.

[27]D. Frias, Análisis de la consistencia interna de las puntuaciones de un instrumento de medida, Valencia: Universidad de Valencia, 2020.

[28]J. L. Ventura, "Tamaño del efecto para la U de Mann-Whitney: aportes al artículo de Valdivia-Peralta et al.,» Revista chilena de neuro-psiquiatría, vol. 54, $\mathrm{n}^{\circ}$ 4, pp. 353-354, 2016.

[29]M. Tomczak y E. Tomczak, «Se revisó la necesidad de informar las estimaciones del tamaño del efecto. Una descripción general de algunas medidas recomendadas del tamaño del efecto,» Trends Sport Sciences, vol. 1, $\mathrm{n}^{\mathrm{o}}$ 21, pp. 19-25, 2014.

[30]S. Domínguez, «Magnitud del efecto, una guía rápida,» Educación Médica, vol. 19, nº 4, pp. 251-254, 2018.

\section{RESUMEN CURRICULAR}

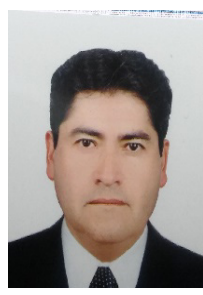

Calizaya López, José Manuel, Doctor en Ciencias de la Educación, Magíster en Ciencias con Mención en Gestión Social - Desarrollo Sostenible y Licenciado en Trabajo Social. Docente Principal y Director del Departamento Académico de Trabajo Social, Docente investigador UNSAINVESTIGA y miembro del Instituto de Investigaciones Sociales de la Universidad Nacional de San Agustín, Arequipa, Perú

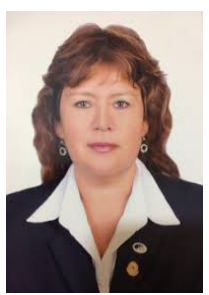

Monzón Alvarez, Gloria Isabel, Doctora en Administración (DBA), Magister en Estrategias de Desarrollo y Políticas Sociales, Licenciada en Trabajo Social, integrante de la Unidad de Investigación de la Facultad de Ciencias Histórico-Sociales. Experta en ejecución de Programas de Bienestar Social, Promoción de la Salud y Políticas del Sistema Integral de Salud Pública. Docente en la Escuela Profesional de Trabajo Social de la Universidad Nacional de San Agustín

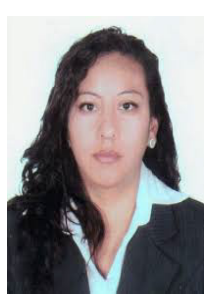

Miaury Vilca, Ana Rosario, Magister en Gerencia Social de la Escuela de Posgrado de la Pontificia Universidad Católica del Perú, con estudios culminados en el Doctorado en Gobernabilidad y Gestión Pública Estratégica; Diplomada en Programa de Gobernabilidad y Gerencia Política The George Washington Univesity The Graduate School Of Political Management y la Corporación Andina de Fomento. Docente del Programa de Sociología de la Universidad Nacional de San Agustín de Arequipa

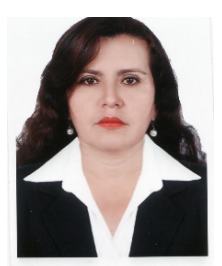

Pinto Pomareda, Hilda Lizbeth, Maestra en Ciencias, con mención en Gerencia Social y de Recursos Humanos, con experiencia en el puesto de Gerente Jefe de Departamento y de Área en la Gestión Pública. Docente Asociado en el programa de estudios de Trabajo Social, delegada de los docentes auxiliares ante la Asamblea Universitaria, Docente investigador UNSA-INVESTIGA

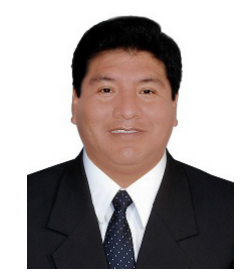

Evangelista Aliaga, José Luis, Maestro en Ciencias: Relaciones Industriales, Especialidad: Gerencia Estratégica de Recursos Humanos, Doctor en Ciencias Empresariales. Docente del Programa de Relaciones Industriales y Públicas Universidad Nacional de San Agustín. Miembro del Instituto de Investigaciones Sociales de la Universidad Nacional de San Agustín, Arequipa, Perú 